\title{
Supporting Information Acid erosion of carbonate fractures and accessibility of arsenic-bearing minerals: In Operando synchrotron-based microfluidics experiment
}

\author{
Hang Deng ${ }^{1, *}$, Jeffrey P. Fitts ${ }^{2}$, Ryan V. Tappero ${ }^{3}$, Julie J. Kim ${ }^{4}$, Catherine A. Peters ${ }^{4}$ \\ 1 Energy Geosciences Division, Lawrence Berkeley National Laboratory, Berkeley, CA, 94720; \\ 2 Columbia Electrochemical Energy Center, Columbia University, New York City, NY, 10027; \\ 3 Photon Sciences Department, Brookhaven National Laboratory, Upton, NY 11973; \\ 4 Department of Civil and Environmental Engineering, Princeton University, Princeton, NJ 08544 \\ *Corresponding author hangdeng@lbl.gov
}

\section{Table of Contents}

1. Microfluidic cell

2. X-ray Attenuation $\quad$ S1

3. X-ray Microspectroscopy $\quad$ S1

4. Powder XRD analyses $\quad$ S2

5. xCT image segmentation $\quad$ S3

6. Quantification of Arsenic Content $\quad$ S3

7. Calculation of diffusion-limited reaction rate $\quad$ S6

$\begin{array}{ll}\text { References } & \text { S7 }\end{array}$

\section{List of figures}

Figure S1. Plan and side view of the microfluidic cell used in the experiment.

Figure S2. Powder XRD patterns of the unreacted (red) and reacted (blue) Eagle Ford shale. Each peak is marked with its corresponding mineral phase; calcite $(\mathrm{C})$, quartz $(\mathrm{Q})$, pyrite $(\mathrm{P})$, and clay minerals $(\mathrm{CM})$.

Both patterns were obtained using a silver anode material (wavelength $=0.55941 \AA$ ).

Figure S3. Histograms of the grayscales of the CT images. The arrows indicate the threshold values used for separating pore-space from minerals (black) and for separating calcite from the rest of the minerals (green). The dashed arrows used thresholding based on visual inspection, and the solid line arrows indicate the threshold values determined by Otsu's method.

Figure S4. Calibration curve of XRF intensities spanning compositions in the pyrite to arsenopyrite solid-

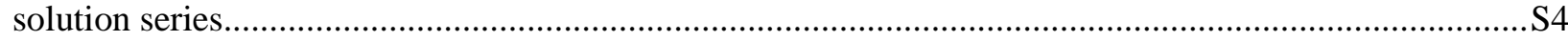

Figure S5. Calibration curve for arsenate $\mathrm{As}(\mathrm{V})$ oxyanion attached to ferrihydrite for various $\mathrm{As} \mathrm{K} \alpha$ to $\mathrm{Fe}$

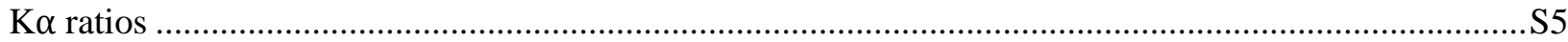

\section{List of tables}

Table S1. Instrumental and compositional parameters for SNRLXRF..................................................S4

Table S2. Chemical compositions considered for As(V)-FHY series .................................................... 55

Table S3. Cubic spline coefficients for XRF intensities of the Pyrite-Arsenopyrite As(-I) substitution

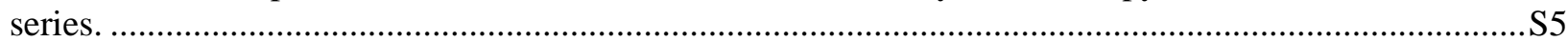

Table S4. Cubic spline coefficients for As(V)-FHY series..............................................................S6 
1. Microfluidic cell

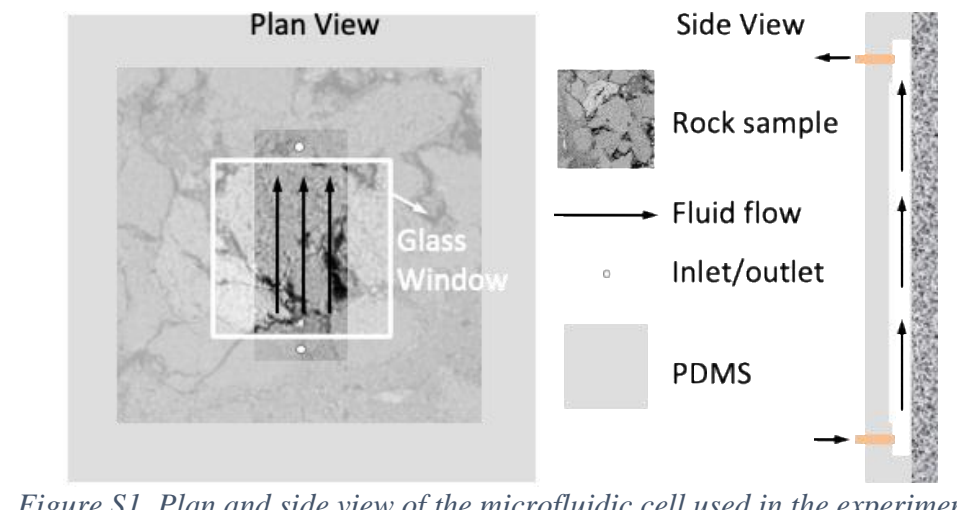

\section{X-ray Attenuation}

The X-ray tomography beamline X2B at the National Synchrotron Light Source (NSLS) was operated as a full-field $\mathrm{X}$-ray microscope with a monochromatic $30 \mathrm{KeV}$ incident $\mathrm{X}$-ray beam defined at $5 \times 5 \mathrm{~mm}$ illumination area by tantalum horizontal and vertical slits. A scintillator converted the transmitted X-ray into visible light and an objective (5x, Zeiss) focused the beam on a $2.56 \mathrm{k} \times 2.16 \mathrm{k}$ pixel CCD camera (PCO Edge) resulting in a $3.3 \times 2.8 \mathrm{~mm}$ field of view. The synchrotron-based X-rays and full-field optical configuration enabled the experiments by providing collimated monochromatic incident energy, high spatial resolution $(1.3 \mu \mathrm{m}$ pixel size), mosaic imaging produced and thus large field of view (12.8 $\mathrm{mm} \times 8.3 \mathrm{~mm}$ in our experiment), and time-series imaging during flow (4 mosaic images/hour).

A dark field (DF) image was collected at the beginning of the flow experiment in order to measure the baseline of every pixel in the CCD camera. In order to normalize the sample attenuated signal, a flat field (FF) image was collected at each time point both before and after collecting the attenuation mosaic. The DF collected at the beginning of the flow experiment was subtracted from every image. Then the full field (FLF) images were divided by the difference image between FF and DF to calculate the transmitted X-ray intensity (I).

\section{X-ray Microspectroscopy}

The XFM beamline (4-BM) at the National Synchrotron Light Source II (NSLS-II) beamline uses Kirkpatrick-Baez (KB) mirrors to deliver focused X-rays (2 - $10 \mathrm{~mm}$ spot) with tunable energy using a $\mathrm{Si}(111)$ double crystal monochromator ( $\mathrm{Si}(220)$ and $\mathrm{Si}(311)$ also available). Sample was oriented $45^{\circ}$ to the incident beam and the XRF detector (Hitachi 7-element Vortex SDD) was positioned $90^{\circ}$ to the incident beam. Images were collected by continuously rastering the sample in the microbeam using a Newport stage with a $20 \mathrm{~mm}$ step size and $50 \mathrm{~ms}$ dwell time per pixel for coarse navigation maps, and a $5 \mathrm{~mm}$ step size and $100 \mathrm{~ms}$ dwell time per pixel for the fine resolution (and valence) maps. Data were processed using LARCH ${ }^{1}$.

Arsenic K-edge micro-X-ray absorption near edge structure ( $\mu$ XANES) spectra were collected in fluorescence mode at 20 points of interest (POIs) over an energy range of 11.85-11.98 keV. Reference $\mu$ XANES spectra of As(III) salt and mineral standards of arsenopyrite, orpiment and ferrihydrite-bound arsenate $(\mathrm{As}(\mathrm{V}))$ were also collected. Linear combination fitting $(\mathrm{LCF})$ of the Arsenic $\mu$ XANES spectra of the 20 POIs in the sample was performed in ATHENA ${ }^{2}$. Criteria 
for adding additional components to the model fits were that the component improved the goodness-of-fit parameter by at least $20 \%$ and represented greater than $5 \%$ of the mixture. The sum was not forced to equal one and negative components were not allowed. Chemical speciation maps were collected within the ROI at four energies: 11.980, 11.874, 11.871 and $11.868 \mathrm{keV}$. A least-squares fit of the images was performed to normalize X-ray absorption coefficient of arsenic standards with the SMAK software package ${ }^{3}$. By integrating the arsenic signal at each valence state, we quantified the relative abundance of different arsenic species.

The configuration used at the hard X-ray microprobe (13-ID-E) at the Advanced Photon Sources (APS) is similar to 4-BM, with the sample oriented $45^{\circ}$ to the incident beam and the XRF detector positioned $90^{\circ}$ to the incident beam. Data collection used a $20 \mu \mathrm{m}$ step size and $20 \mathrm{~ms}$ dwell time per pixel, at an incident energy of $18.000 \mathrm{keV}$. Element maps of this thin section were generated using $\mathrm{LARCH}^{1}$.

\section{Powder XRD analyses}

Reacted and unreacted samples of the Eagle Ford shale were powdered for X-ray diffraction analyses. Bruker D8 Advance Diffractometer was set up with a Ag X-ray source, divergence and soller slit sizes of $1.2 \mathrm{~mm}$ and $2.5 \mathrm{~mm}$, respectively, and measurements were taken with a step size of $0.025^{\circ}$. Mineral phases were identified and matched using the Diffrac.EVA software and PDF database from ICDD. Three replicates were analyzed for each of the unreacted and reacted samples, with no significant differences in the peaks and their locations. Figure S2 shows one pattern from unreacted and one pattern from the reacted sample.

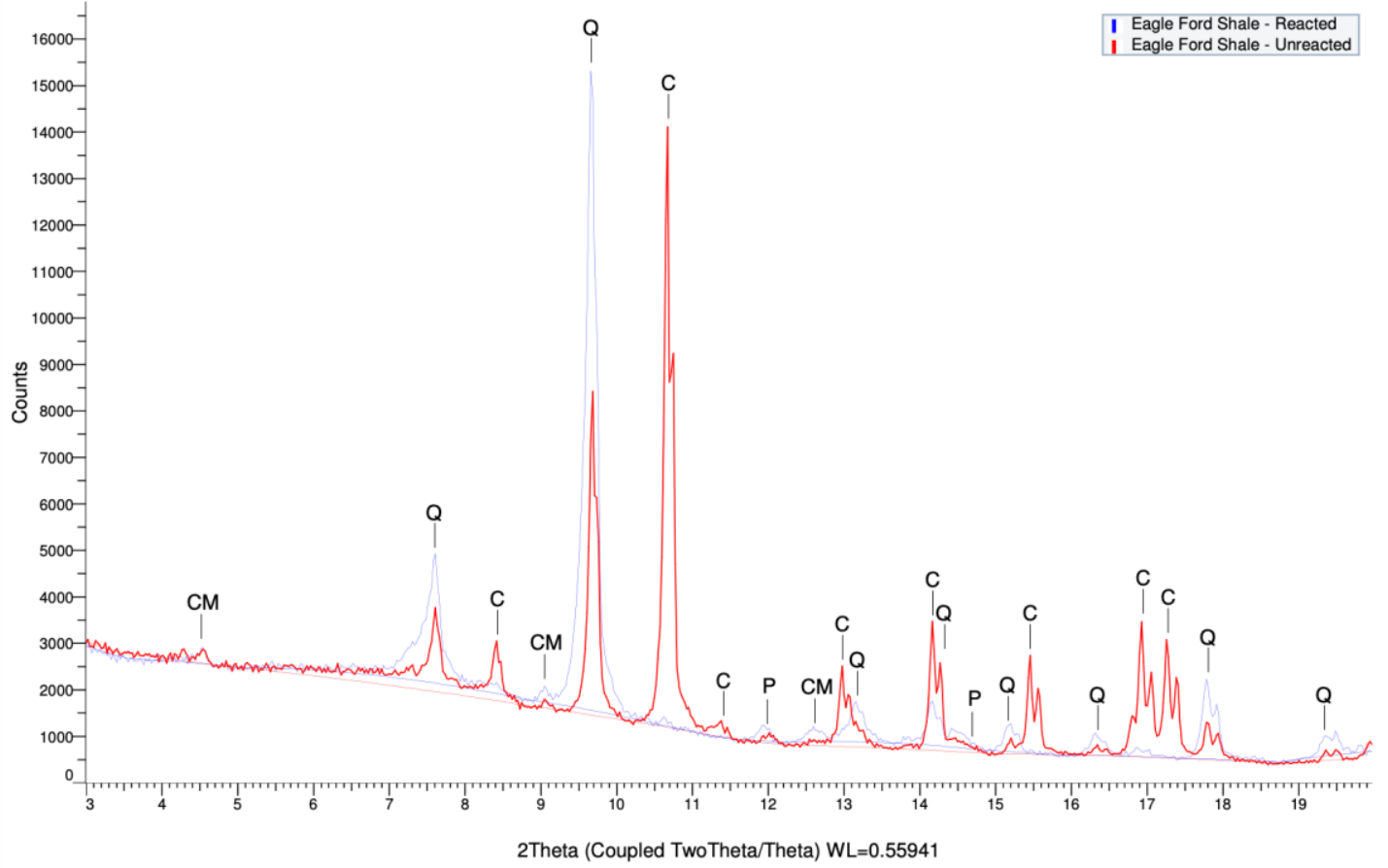

Figure S2. Powder XRD patterns of the unreacted (red) and reacted (blue) Eagle Ford shale. Each peak is marked with its corresponding mineral phase; calcite $(C)$, quartz $(Q)$, pyrite $(P)$, and clay minerals $(C M)$. Both patterns were obtained using a silver anode material (wavelength $=0.55941 \AA$ ).

Quartz, calcite, pyrite, and some clay minerals were identified in the unreacted portion of the Eagle Ford shale. Calcite peaks are missing in the pattern obtained from the reacted shale sample, indicating near depletion of calcite post reaction. 


\section{5. xCT image segmentation}

The thresholding method was used to separate different phases, i.e. the pore space versus the mineral phases, and calcite versus other mineral phases using Matlab. This is feasible because of the distinctly different peaks in the grayscale values corresponding to each phase or class in the histogram, as shown in Figure S3. Quantifying uncertainties associated with image processing has been one paramount challenge because of the lack of ground truth. In our study, to provide one way of quantifying uncertainties associated with thresholding and the estimates of porosity and surface area, threshold values determined from two methods were used for the segmentation. One is based on visual inspection and corresponds to the minimum of the overlapping grayscale peaks (dashed arrows), and the other is determined by Otsu's method that minimizes inter-class variances (solid line arrows).

The porosity is determined as the ratio between the number of voxels classified as pore space to the number of voxels in the volume analyzed. This may underestimate the porosity for pore space below the image resolution is not considered. The surface area is identified by applying the bwperim filter in Matlab 2019a to track the perimeter pixels of the solid phases in each 2D slice, and the bulk surface area is calculated as the ratio between the summation of the surface area and the corresponding volume analyzed.

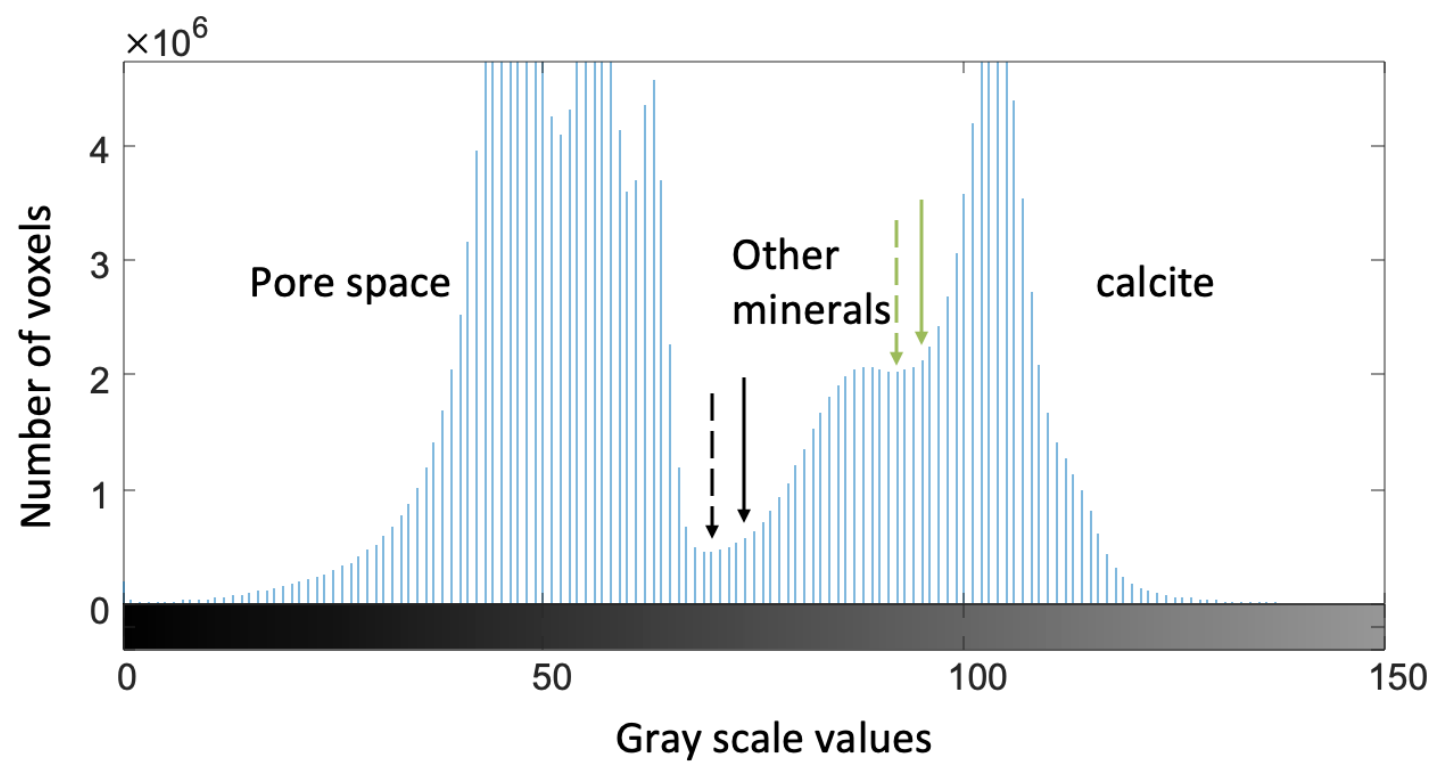

Figure S3. Histograms of the grayscales of the CT images. The arrows indicate the threshold values used for separating porespace from minerals (black) and for separating calcite from the rest of the minerals (green). The dashed arrows used thresholding based on visual inspection, and the solid line arrows indicate the threshold values determined by Otsu's method.

\section{Quantification of Arsenic Content}

NIST Synchrotron Naval Research Laboratory computer program for quantitative X-ray fluorescence analysis (SNRLXRF) ${ }^{4}$ was used to determine the theoretical fluorescence intensities of $\mathrm{Fe} \mathrm{K} \alpha$ and $\mathrm{As} \mathrm{K} \alpha$ for various As-Pyrite compositions, ranging from pure pyrite to arsenopyrite. Similarly, a theoretical intensity curve for various As wt. \% was generated for Arsenic associated with ferrihydrite (FHY), assuming the mechanism of arsenic presence to be via adsorption. Using the "predict" mode in SNRLXRF, instrumental parameters such as the detector filter thicknesses, incident energy, detector and sample configuration, as well as compositional parameters such as 
sample density and thickness were considered to increase accuracy of the quantification. For the As-pyrite solid solution series, the densities were interpolated between the densities of pyrite and arsenopyrite, while a constant density was assumed for the As-FHY series. Table S1 summarizes all inputs to SNRLXRF used in common for both series.

Table S1. Instrumental and compositional parameters for SNRLXRF

\begin{tabular}{cc}
\hline Parameter & Value \\
\hline Detector Filter: Aluminum & $64 \mu \mathrm{m}$ \\
Detector Filter: Air & $75 \mathrm{~mm}$ \\
Sample Thickness & $30 \mu \mathrm{m}$ \\
Angles in \& out & $45^{\circ} \& 135^{\circ}$ \\
Incident Energy & $12 \mathrm{keV}$ \\
\hline
\end{tabular}

Using the calibration points of the theoretical emission intensities and based on cubic spline fitting, measured emission intensities were interpreted to find the approximate arsenic weight percentage incorporated in the solid. Two calibration curves for arsenian-pyrite and As(V)-FHY are presented in Figures S4 and S5, respectively.

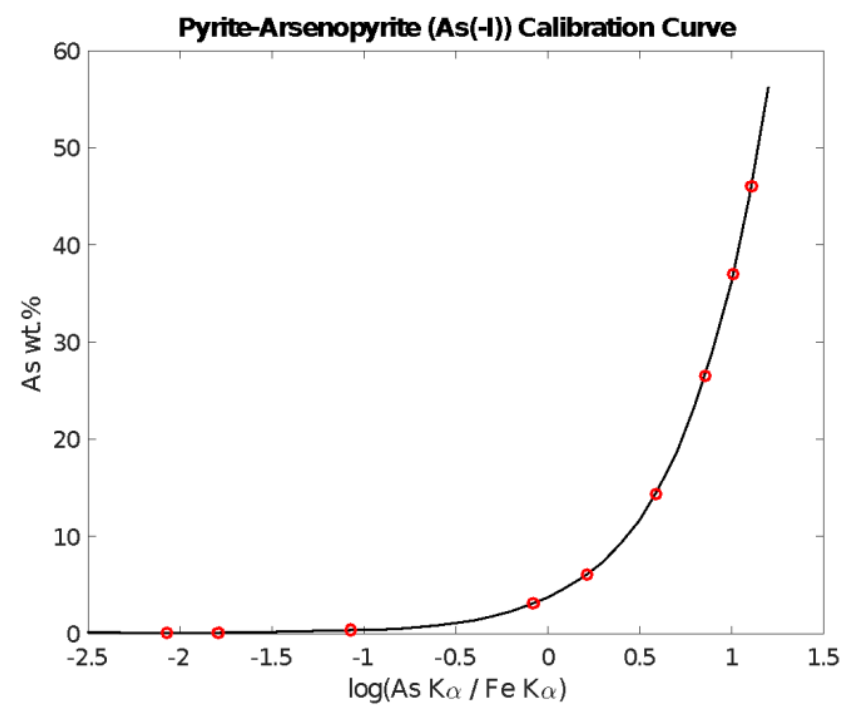

Figure S4. Calibration curve of XRF intensities spanning compositions in the pyrite to arsenopyrite solid-solution series. 


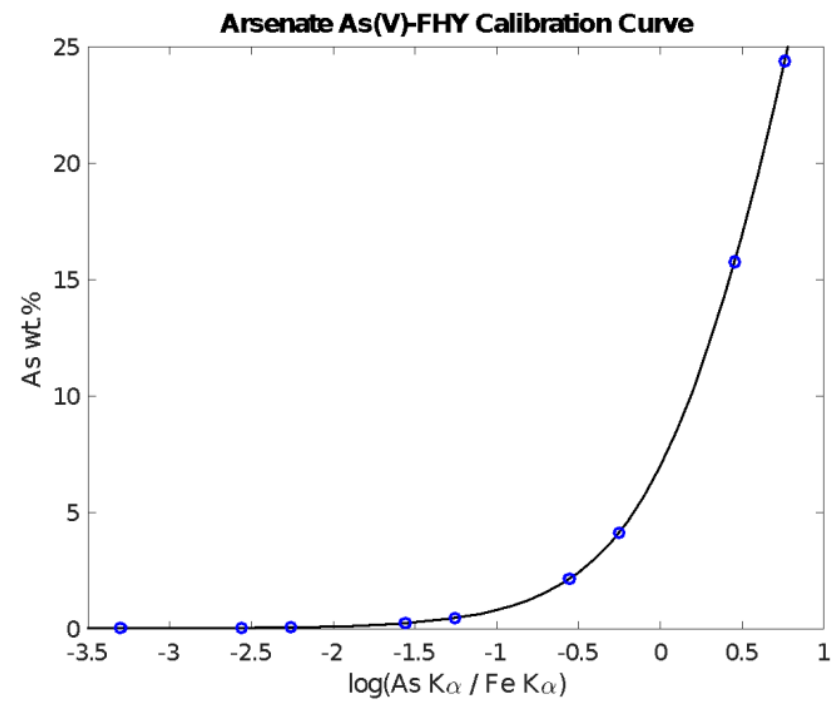

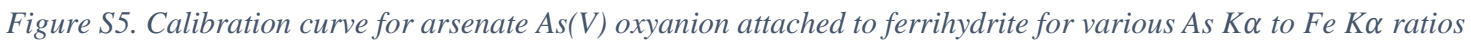

Table S2 summarizes the chemical formula of each point on the As(V)-FHY calibration curve (Figure S5). Chemical compositions corresponding to more than a 1:1 ratio of ferrihydrite:arsenate were not considered as they are not likely to exist under the relevant conditions.

Table S2. Chemical compositions considered for As(V)-FHY series

\begin{tabular}{|c|c|c|c|c|c|c|c|c|}
\hline $\mathrm{Fe}_{2} \mathrm{O}_{3^{-}}$ & $\mathrm{Fe}_{2} \mathrm{O}_{3^{-}}$ & $\mathrm{Fe}_{2} \mathrm{O}_{3^{-}}$ & $\mathrm{Fe}_{2} \mathrm{O}_{3^{-}}$ & $\mathrm{Fe}_{2} \mathrm{O}_{3^{-}}$ & $\mathrm{Fe}_{2} \mathrm{O}_{3^{-}}$ & $\mathrm{Fe}_{2} \mathrm{O}_{3^{-}}$ & $\mathrm{Fe}_{2} \mathrm{O}_{3^{-}}$ & $\mathrm{Fe}_{2} \mathrm{O}_{3^{-}}$ \\
$0.5 \mathrm{H}_{2} \mathrm{O}+$ & $0.5 \mathrm{H}_{2} \mathrm{O}+$ & $0.5 \mathrm{H}_{2} \mathrm{O}+$ & $0.5 \mathrm{H}_{2} \mathrm{O}+$ & $0.5 \mathrm{H}_{2} \mathrm{O}+$ & $0.5 \mathrm{H}_{2} \mathrm{O}+$ & $0.5 \mathrm{H}_{2} \mathrm{O}+$ & $0.5 \mathrm{H}_{2} \mathrm{O}+$ & $0.5 \mathrm{H}_{2} \mathrm{O}+$ \\
$0.0001\left(\mathrm{AsO}_{4}\right)$ & $0.0005\left(\mathrm{AsO}_{4}\right)$ & $0.001\left(\mathrm{AsO}_{4}\right)$ & $0.005\left(\mathrm{AsO}_{4}\right)$ & $0.01\left(\mathrm{AsO}_{4}\right)$ & $0.05\left(\mathrm{AsO}_{4}\right)$ & $0.1\left(\mathrm{AsO}_{4}\right)$ & $0.5\left(\mathrm{AsO}_{4}\right)$ & $\mathrm{AsO} \mathrm{O}_{4}$ \\
\hline
\end{tabular}

Equation S1 is the general polynomial expression used to generate Figures S4 and S5, and Tables S3 and S4 summarize the corresponding coefficients in the As(-I) and As(V) series, respectively, for all relevant intervals considered. The general polynomial equation can be written as,

$$
f(x)=a\left(x-x_{1}\right)^{3}+b\left(x-x_{1}\right)^{2}+c\left(x-x_{1}\right)+d
$$

where a,b,c,d are the polynomial coefficients defined for each [x1 x2].

Table S3. Cubic spline coefficients for XRF intensities of the Pyrite-Arsenopyrite As(-I) substitution series.

\begin{tabular}{ccccc}
\hline $\begin{array}{c}\log (\text { As K } \alpha / \mathrm{FeK} \alpha) \\
\text { Interval }\end{array}$ & $\mathrm{a}$ & $\mathrm{b}$ & $\mathrm{c}$ & $\mathrm{d}$ \\
\hline$[-2.07-1.79]$ & -0.129 & 0.430 & -0.021 & 0.031 \\
{$[-1.79-1.07]$} & -0.129 & 0.322 & 0.189 & 0.056 \\
{$[-1.07-0.08]$} & 2.317 & 0.044 & 0.453 & 0.312 \\
{$[-0.080 .21]$} & 8.591 & 6.941 & 7.383 & 3.067
\end{tabular}


[0.21 0.59$]$

21.897

14.467

13.634

6.029

$[0.590 .86]$

16.284

39.101

33.721

14.330

[0.86 1.01]

89.937

52.193

58.188

26.489

[1.01 1.11]

89.937

93.475

80.475

36.936

Table S4. Cubic spline coefficients for As(V)-FHY series

\begin{tabular}{ccccc}
\hline $\begin{array}{c}\log (\text { As K } \alpha / \mathrm{FeK} \alpha) \\
\text { Interval }\end{array}$ & $\mathrm{a}$ & $\mathrm{b}$ & $\mathrm{c}$ & $\mathrm{d}$ \\
\hline$[-3.30-2.56]$ & 0.016 & 0.019 & 0.002 & 0.004 \\
{$[-2.56-2.26]$} & 0.016 & 0.054 & 0.055 & 0.022 \\
{$[-2.26-1.56]$} & 0.227 & 0.068 & 0.092 & 0.044 \\
{$[-1.56-1.25]$} & 0.428 & 0.545 & 0.522 & 0.220 \\
{$[-1.25-0.55]$} & 1.603 & 0.934 & 0.970 & 0.440 \\
{$[-0.55-0.25]$} & 6.031 & 4.307 & 4.646 & 2.133 \\
{$[-0.250 .46]$} & 1.121 & 9.788 & 8.915 & 4.103 \\
{$[0.460 .76]$} & 1.121 & 12.169 & 24.471 & 15.730 \\
\hline
\end{tabular}

\section{Calculation of diffusion-limited reaction rate}

According to ${ }^{5}$, the diffusion-limited reaction rate can be estimated based on the diffusive flux across the fracture surface

$R_{d}=D_{A L}\left(C_{s}-C_{b}\right) / L_{A L}$ eqn(S2) where $C_{s}$ and $C_{b}$ are the concentrations of $\mathrm{Ca}^{2+}$ at the reaction front and in the bulk fluid in the fracture channel, respectively, and $D_{A L}$ is the effective diffusion coefficient and is typically calculated from Archie's law

$D_{A L}=D \phi^{m}$

eqn(S3)

where $m$ is the cementation factor and is set to be 2, which is a typical value reported for various rocks ${ }^{6}$. Using a molecular diffusion coefficient $(D)$ of $1 \times 10^{-9} \mathrm{~m}^{2} / \mathrm{s}$, the effective diffusion coefficient is $3.6 \times 10^{-10} \mathrm{~m}^{2} / \mathrm{s}$ for a porosity of $60 \%$. At the $\mathrm{pH}$ of our experimental system, the equilibrium $\mathrm{Ca}^{2+}$ concentration is $4.0 \times 10^{-3} \mathrm{~mol} / \mathrm{L}$, which provides an upper boundary for $C_{S}$. Assuming local equilibrium at the mineral surface due to the fast kinetic rate of calcite dissolution, for an altered layer thickness of $100 \mu \mathrm{m}$, the diffusion-limited reaction rate is on the order of $1 \times 10^{-5} \mathrm{~mol} / \mathrm{m}^{2} \mathrm{~s}$, which is comparable to the measured instantaneous reaction rate at hour 24. 


\section{References}

(1) Newville, M. (2013). Larch: An Analysis Package for XAFS and Related Spectroscopies. In Journal of Physics: Conference Series (Vol. 430, p. 012007). https://doi.org/10.1088/1742$\underline{6596 / 430 / 1 / 012007}$

(2) Ravel, B., \& Newville, M. (2005). ATHENA, ARTEMIS, HEPHAESTUS: data analysis for $\mathrm{X}$-ray absorption spectroscopy using IFEFFIT. In Journal of Synchrotron Radiation (Vol. 12, Issue 4, pp. 537-541). https://doi.org/10.1107/s0909049505012719

(3) Webb, S. M. (2006). SMAK: Sam's Microprobe Analysis Kit. Stanford Synchrotron Radiation Laboratory, 1.64.

(4) Birks, L. S., Gilfrich, J. V., \& Criss, J. W. (1977). NRLXRF, A fortran program for X-ray fluorescence analysis: users'guide. Naval Research Laboratory, Washington, DC.

(5) Deng, H., Molins, S., Steefel, C., DePaolo, D., Voltolini, M., Yang, L., \& Ajo-Franklin, J. (2016). A 2.5D Reactive Transport Model for Fracture Alteration Simulation. Environmental Science \& Technology, 50(14), 7564-7571.

(6) Cho, Y., Eker, E., Uzun, I., Yin, X., \& Kazemi, H. (2016). Rock Characterization in Unconventional Reservoirs: A Comparative Study of Bakken, Eagle Ford, and Niobrara Formations. In SPE Low Perm Symposium. https://doi.org/10.2118/180239-ms 